REPRESENTATION THEORY

An Electronic Journal of the American Mathematical Society

Volume 13, Pages 19-32 (February 13, 2009)

S 1088-4165(09)00341-0

\title{
MIRKOVIĆ-VILONEN CYCLES AND POLYTOPES FOR A SYMMETRIC PAIR
}

\author{
JIUZU HONG
}

\begin{abstract}
Let $G$ be a connected, simply-connected, and almost simple algebraic group, and let $\sigma$ be a Dynkin automorphism on $G$. Then $\left(G, G^{\sigma}\right)$ is a symmetric pair. In this paper, we get a bijection between the set of $\sigma$ invariant MV cycles (polytopes) for $G$ and the set of MV cycles (polytopes) for $G^{\sigma}$, which is the fixed point subgroup of $G$; moreover, this bijection can be restricted to the set of MV cycles (polytopes) in irreducible representations. As an application, we obtain a new proof of the twining character formula.
\end{abstract}

\section{INTRODUCTION}

Let $G$ be a connected semisimple algebraic group over $\mathbb{C}$, and let $\mathcal{G}$ be the affine Grassmannian of $G$. Let $\mathcal{G}_{\lambda}$ be the $G(\mathbb{C}[[t]])$-orbit on $\mathcal{G}$ corresponding to a dominant coweight $\lambda$ on $G$. Let $I C_{\lambda}$ be the spherical perverse sheaf supported on $\overline{\mathcal{G}_{\lambda}}$. V. Ginzburg [G] and Mirković and Vilonen [MV] set up the geometric Satake correspondence, which says that the category of spherical perverse sheaves on $\mathcal{G}$ is equivalent to the category of finite dimensional representations of the Langlands dual group $G^{\vee}$ of $G$; in particular, the irreducible representation $V(\lambda)$ of $G^{\vee}$ with highest weight $\lambda$ is identified with the cohomology group $H^{*}\left(\mathcal{G}, I C_{\lambda}\right)$. Furthermore, Mirković and Vilonen [MV] discovered Mirković-Vilonen cycles which affords a natural basis of $V(\lambda)$.

In $\mathrm{A}$, Anderson studied the moment polytopes of Mirković-Vilonen cycles, which are called Mirković-Vilonen polytopes, and showed that these polytopes could be used to understand the combinatorics of representations of $G^{\vee}$. In K1, Kamnitzer gave an explicit combinatorial description of the MV cycles and polytopes. He showed that canonical basis and MV cycles are governed by the same combinatorics, i.e., MV cycles $\longleftrightarrow$ MV polytopes $\longleftrightarrow$ canonical basis, are bijections.

Let $\sigma$ be a nontrivial Dynkin automorphism of $G$. We have a Dynkin automorphism on $G^{\vee}$ induced from $\sigma$. Let $G^{\sigma}$ be the identity component of a fixed point group of $\sigma$ on $G$. Let $\lambda$ be a $\sigma$-invariant dominant coweight of $G$, which can also be viewed as a dominant coweight of $G^{\sigma}$. Let $v(\lambda)$ be the irreducible representation of $G^{\vee}$ with highest weight $\lambda$. We have a natural action of $\sigma$ on $V(\lambda)$ induced from the action of the automorphism on $G^{\vee}$, which fixes the highest weight vector in $V(\lambda)$. For a $\sigma$-invariant coweight $\mu$ for $G, \sigma$ acts on the weight space $V_{\mu}(\lambda)$. The twining character $\operatorname{ch}^{\sigma} V(\lambda)$ is defined to be $\sum_{\sigma(\mu)=\mu} \operatorname{trace}\left(\left.\sigma\right|_{V_{\mu}(\lambda)}\right) e^{\mu}$. It is related to the character of the irreducible representation of $\left(G^{\sigma}\right)^{\vee}$ with highest weight $\lambda$ through the twining character formula, which is attributed to Jantzen [J] under the name

Received by the editors May 13, 2008 and, in revised form, November 15, 2008

2000 Mathematics Subject Classification. Primary 20G05; Secondary 14M15.

(C)2009 American Mathematical Society 
of Jantzen theorem in $\mathrm{KLP}$. Though there are many proofs in the literature (for example, [J], $[\mathbf{N},[\mathrm{KLP})$, it seems that there is no satisfactory explanation for why Langlands dual appears in this formula.

In this paper, we consider the action of $\sigma$ on MV cycles and MV polytopes. The main result of the paper is to give an explicit bijection between $\sigma$-invariant MV cycles (polytopes) for $G$ to MV cycles (polytopes) for $G^{\sigma}$. In terms of polytopes, it sends $\sigma$-invariant MV polytopes $P$ for $G$, to $P^{\sigma}$, which is a MV polytope for $G^{\sigma}$. The bijection can be restricted to MV cyles (polytopes) in irreducible representation space.

In this paper, we also show that the automorphism on $G^{\vee}$ from Tannakian formalism is a Dynkin automorphism. On $V(\lambda)$, there are two actions of $\sigma$, where one is induced from $G^{\vee}$, and the other one is induced from the action of $\sigma$ on MV cycles. We show that both of them agree, then we get a new proof of twining character formula through geometric Satake correspondence.

Acknowledgments. I would like to thank Professor T. Tanisaki for his contributions to the paper, including ideas, discussions and useful comments; I also appreciate his careful reading and enormous help on the improvement of writing. I am very indebted to Professor N. Xi for his support and encouragement on my study of mathematics, and also for his help with this paper. Finally, I would like to thank Professor G. Lusztig for some beneficial conversations during his stay in China in July, 2007.

This paper was written during the author's visit to Hebrew University in Jerusalem under the support of the Marie Curie Research Training Network. During the writing of this paper, I heard that Professor S. Naito and D. Sagaki have given a closely related result almost at the same time [NS1], [NS2].

I would also like to thank the referee for very helpful comments.

\section{DYNKIN AUTOMORPHISM}

2.1. Notations. Let $G$ be a connected, simply-connected and almost simple algebraic group of rank $\ell$ over $\mathbb{C}$. Let $T$ be a maximal torus of $G$ and let $X^{*}=$ $\operatorname{Hom}\left(T, \mathbb{C}^{\times}\right), X_{*}=\operatorname{Hom}\left(\mathbb{C}^{\times}, T\right)$ denote the weight and coweight lattices of $T$. Then we have a natural perfect pairing $\langle\rangle:, X_{*} \times X^{*} \rightarrow \mathbb{Z}$. Let $W=N(T) / T$ denote the Weyl group.

Let $I=\{1, \cdots, l\}$ denote vertices of the Dynkin diagram of $G$. Let $B$ be a Borel subgroup of $G$ containing $T$. Let $\alpha_{1}, \alpha_{2}, \cdots, \alpha_{l}$ and $\alpha_{1}^{\vee}, \alpha_{2}^{\vee}, \cdots, \alpha_{l}^{\vee}$ be simple roots and simple coroots of $G$ with respect to $B$, respectively. Then $a_{i j}=\left\langle\alpha_{i}^{\vee}, \alpha_{j}\right\rangle$ is the entry of the Cartan matrix of $G$. Note that $\left(X_{*}, X^{*},\langle\rangle,, \alpha_{i}^{\vee}, \alpha_{i} ; i \in I\right)$ is the root datum of $G$. Let $\lambda_{1}, \cdots, \lambda_{l} \in X^{*} \otimes \mathbb{R}$ be fundamental weights.

For $i \in I$, let $x_{i}: \mathbb{C} \rightarrow G$ and $y_{i}: \mathbb{C} \rightarrow G$ be root homomorphisms (corresponding to $\alpha_{i}$ and $-\alpha_{i}$, respectively) which together with $T, B$ form a pinning of $G$.

Let $s_{1}, \cdots, s_{\ell} \in W$ be the set of simple reflections. Let $w_{0}$ be the longest element of $W$, and let $m$ be its length.

We use $\geq$ for the usual partial order on $X_{*}$, so that $\mu \geq \nu$ if and only if $\mu-\nu$ is a sum of positive coroots. More generally, for each $w \in W$, we have the twisted partial order $\geq_{w}$ on $X_{*}$, where $\mu \geq_{w} \nu$ if and only if $w^{-1} \cdot \mu \geq w^{-1} \cdot \nu$.

A reduced word for an element $w \in W$ is a sequence of indices $\mathbf{i}=\left(i_{1}, \cdots, i_{k}\right)$ $\in I^{k}$ such that $w=s_{i_{1}} \cdot s_{i_{2}} \cdots s_{i_{k}}$ is a reduced expression. In this paper, a reduced 
word will always mean a reduced word for $w_{0}$, where $w_{0}$ is the longest element in $W$.

2.2. Group structure of $G^{\sigma}$. Let $\sigma: I \rightarrow I$ be a nontrivial bijection, satisfying $a_{\sigma(i) \sigma(j)}=a_{i j}$ for all $i, j \in I$. We assume that there are automorphisms $\sigma: X^{*} \rightarrow$ $X^{*}$ and $\sigma: X_{*} \rightarrow X_{*}$ of Z-modules satisfying $\sigma\left(\alpha_{i}\right)=\alpha_{\sigma(i)}$ and $\sigma\left(\alpha_{i}^{\vee}\right)=\alpha_{\sigma(i)}^{\vee}$ for any $i \in I$. Then $\sigma$ induces an automorphism $\sigma: G \rightarrow G$ of algebraic groups, such that $\sigma\left(x_{i}(a)\right)=x_{\sigma(i)}(a)$ and $\sigma\left(y_{i}(a)\right)=y_{\sigma(i)}(a)(\forall i \in I)$. We call $\sigma$ a Dynkin automorphism on $G$. In particular, we have $\sigma(B)=B$ and $\sigma(T)=T$.

Let $G^{\sigma}$ be the fixed point group of $\sigma$ on $G$, and let $T^{\sigma}$ and $B^{\sigma}$ be the fixed point groups of $T$ and $B$, respectively. Then $G^{\sigma}, B^{\sigma}$ and $T^{\sigma}$ are connected; moreover, $G^{\sigma}$ is almost simple algebraic group, under our assumptions on $G$ (see [ST]). We call $\left(G, G^{\sigma}\right)$ a symmetric pair.

We set $X_{*}^{\sigma}=\left\{\lambda \in X_{*} \mid \sigma(\lambda)=\lambda\right\}$, and $X_{\sigma}^{*}=\operatorname{Hom}\left(X_{*}^{\sigma}, \mathbb{Z}\right)$. We have a perfect pairing $X_{*}^{\sigma} \times X_{\sigma}^{*} \rightarrow \mathbb{Z}$ denoted again by $\langle$,$\rangle . Let I_{\sigma}$ be the set of $\sigma$-orbits on $I$.

For any $\eta \in I_{\sigma}$, let $\alpha_{\eta}^{\vee}=2^{h} \sum_{i \in \eta} \alpha_{i}^{\vee} \in X_{*}^{\sigma}$, where $h$ is the number of unordered pairs $(i, j)$ such that $i, j \in \eta, \alpha_{i}+\alpha_{j} \in \Phi$. Note that $h=1$, if $\eta=\{i, j\}$ and $a_{i j}=$ $-1 ; h=0$, otherwise. Let $\theta: X^{*} \otimes \mathbb{R} \rightarrow X_{\sigma}^{*} \otimes \mathbb{R}$ be the natural surjection induced from the perfect pairing $\langle\rangle:, X_{*} \times X^{*} \rightarrow \mathbb{Z}$. Set $\alpha_{\eta}=\theta\left(\alpha_{i}\right)$, and $\lambda_{\eta}=\frac{1}{h} \theta\left(\lambda_{i}\right)$, where $i$ is any element of $\eta$. We have the following proposition (see [KLP], $[\mathrm{J}]$ ).

Proposition 2.1. $\left(X_{*}^{\sigma}, X_{\sigma}^{*}, \alpha_{\eta}^{\vee}, \alpha_{\eta}\right)$ is a root datum of $G^{\sigma}$.

Define $x_{\eta}=\prod_{i \in \eta} x_{i}: \mathbb{C} \rightarrow G^{\sigma}$, by $x_{\eta}(a)=\prod_{i \in \eta} x_{i}(a)$, if $\eta$ has only one element, or $\forall i, j \in \eta$, with $i \neq j, a_{i j}=0$; define $x_{\eta}: \mathbb{C} \rightarrow G^{\sigma}$, by $x_{\eta}(a)=x_{i}(a) x_{j}(2 a) x_{i}(a)$, if $\eta=\{i, j\}, a_{i j}=-1$. We have the following lemma (see [L1]).

Lemma 2.2. Let $x_{1}, x_{2}$ be two simple root subgroup homomorphisms of $G$ of type $A_{2}$ corresponding to $\alpha_{1}$ and $\alpha_{2}$. Then we have

$$
x_{1}\left(a_{1}\right) x_{2}\left(a_{2}\right) x_{1}\left(a_{3}\right)=x_{2}\left(\frac{a_{2} a_{3}}{a_{1}+a_{3}}\right) x_{1}\left(a_{1}+a_{3}\right) x_{2}\left(\frac{a_{1} a_{2}}{a_{1}+a_{3}}\right) .
$$

From this lemma, we see easily that $x_{\eta}$ is a group homomorphism. Similarly, we can define $y_{\eta}$, so that $x_{\eta}$ and $y_{\eta}$ are homomorphisms from $\mathbb{C}$ to $G^{\sigma}$. Since $t x_{\eta}(a) t^{-1}=x_{\eta}\left(\alpha_{\eta}(t) a\right), x_{\eta}$ is a root subgroup homomorphism of $G^{\sigma}$ with root $\alpha_{\eta}$. We have

Proposition 2.3. $\left(T^{\sigma}, B^{\sigma}, x_{\eta}, y_{\eta} ; \eta \in I_{\sigma}\right)$ form a pinning of $G^{\sigma}$.

Clearly, $\sigma: G \rightarrow G$ induces an automorphism of $W$ denoted again by $\sigma$, satisfying $\sigma\left(s_{i}\right)=s_{\sigma(i)}$ for any $i \in I$. Let $W^{\sigma}=\{w \in W \mid \sigma(w)=w\}$. For any $\eta \in I_{\sigma}$ we define $s_{\eta} \in W^{\sigma}$ to be the longest element in the subgroup of $W$ generated by $\left\{s_{i} ; i \in \eta\right\}$. It is known that $W^{\sigma}$ is a Coxeter group on the generators $\left\{s_{\eta} ; \eta \in I_{\sigma}\right\}$. Any element $w \in W^{\sigma}$ can be restricted to $X_{*}^{\sigma}$. Under this restriction, we can see that $W^{\sigma}$ is identified with the Weyl group of $G^{\sigma}$. For $w \in W^{\sigma}$, we denote by $\ell_{\sigma}(w)$ the length of $w$ in the Coxeter group $W^{\sigma}$.

\section{MV CYCLES AND MV POLYTOPES FOR THE SYMMETRIC PAIR}

3.1. Action of $\sigma$ on affine Grassmannian. Let $\mathcal{O}=\mathbb{C}[[t]]$, and let $\mathcal{K}$ be the quotient field of $\mathcal{O}$. Let $\mathcal{G}$ and $\mathcal{G}_{\sigma}$ be affine Grassmannian of $G$ and $G^{\sigma}$, respectively. As the sets of rational points over $\mathbb{C}, \mathcal{G}=G(\mathcal{K}) / G(\mathcal{O})$, and $\mathcal{G}_{\sigma}=G(\mathcal{K})^{\sigma} / G(\mathcal{O})^{\sigma}$. A coweight $\mu \in X_{*}$ gives a point in $\mathcal{G}$, denoted by $\underline{t}^{\mu}$. It is known that $\underline{t}^{\mu}$ is a fixed 
point for the action of $T$ on $\mathcal{G}$. In fact, all the fixed points of $T$ are given in this way.

For a given dominant coweight $\lambda$, we set $\mathcal{G}^{\lambda}=G(\mathcal{O}) \cdot \underline{t}^{\lambda}$. We have the decomposition $\mathcal{G}=\bigsqcup_{\lambda \in X_{*}^{+}} \mathcal{G}^{\lambda}$, where $X_{*}^{+}$is the set of dominant coweights.

Let $N$ be the unipotent radical of $B$. For $w \in W$, we set $N_{w}=w N w^{-1}$. For $w \in W$ and $\mu \in X_{*}$, define the semi-infinite cells by $S_{w}^{\mu}=N_{w}(\mathcal{K}) \cdot \underline{t}^{\mu}$. For simplicity, we set $S^{\mu}=S_{e}^{\mu}=N(\mathcal{K}) \cdot \underline{t}^{\mu}$. We have $\mathcal{G}=\bigsqcup_{\mu \in X_{*}} S^{\mu}$. The semi-infinite cells have the simple containment relation, $\overline{S_{w}^{\mu}}=\bigsqcup_{\nu \leq_{w} \mu} S_{w}^{\nu}$. We see that if $S_{w}^{\mu} \cap S_{v}^{\nu} \neq \varnothing$, then $\nu \leq_{w} \mu$.

We have the closed embedding $\iota: \mathcal{G}_{\sigma} \hookrightarrow \mathcal{G}$. Since $\sigma\left(S^{\lambda}\right)=S^{\sigma(\lambda)}$, we have $\mathcal{G}^{\sigma}=\bigsqcup_{\lambda \in X_{*}^{\sigma}}\left(S^{\lambda}\right)^{\sigma}$.

Set $\left.U:=\left\{g\left(t^{-1}\right) \in G\left(\mathbb{C}\left[t^{-1}\right]\right) \mid g(0)=1\right)\right\}$. Then the fixed point set $U^{\sigma}=$ $\left.\left\{g\left(t^{-1}\right) \in G^{\sigma}\left(\mathbb{C}\left[t^{-1}\right]\right) \mid g(0)=1\right)\right\}$. For a coweight $\lambda$, set $S(\lambda):=N\left(\mathbb{C}\left[t, t^{-1}\right]\right) \cap$ $t^{\lambda} U t^{-\lambda}$ and $S_{\sigma}(\lambda):=N^{\sigma}\left(\mathbb{C}\left[t, t^{-1}\right]\right) \cap t^{\lambda} U^{\sigma} t^{-\lambda}$.

The following result is well known.

Lemma 3.1. Let $\lambda \in X_{*}$. Then the group $S(\lambda)$ acts simply-transitively on $S^{\lambda}$, i.e., $S(\lambda) \simeq S^{\lambda}$, with the map $g \mapsto g . t^{\lambda}$.

Proposition 3.2. The fixed point subvariety of the action of $\sigma$ on $\mathcal{G}$ is exactly identified with $\mathcal{G}_{\sigma}$.

Proof. From Lemma 3.1, we are reduced to showing that $S(\lambda)^{\sigma}=S_{\sigma}(\lambda)$ for $\lambda \in X_{*}^{\sigma}$, and it is easy to see, since

$$
S(\lambda)^{\sigma}=N\left(\mathbb{C}\left[t, t^{-1}\right]\right)^{\sigma} \cap\left(t^{\lambda} U t^{-\lambda}\right)^{\sigma}=N^{\sigma}\left(\mathbb{C}\left[t, t^{-1}\right]\right) \cap t^{\lambda} U^{\sigma} t^{-\lambda}=S_{\sigma}(\lambda) .
$$

From $\overline{\mathcal{G}^{\lambda}}=\bigsqcup_{\mu \leq \lambda} \mathcal{G}^{\mu}, \overline{S_{w}^{\mu}}=\bigsqcup_{\nu \leq_{w} \mu} S_{w}^{\nu}$ and the above proposition, we can easily see that

Corollary 3.3. For $\lambda$ a $\sigma$-invariant, and $w$ a $\sigma$-invariant element in $W$, we have $\left(\mathcal{G}^{\lambda}\right)^{\sigma}=\mathcal{G}_{\sigma}^{\lambda}, \overline{\mathcal{G}}^{\alpha}=\overline{\mathcal{G}_{\sigma}^{\lambda}},\left(S_{w}^{\mu}\right)^{\sigma}=\left(S_{\sigma}\right)_{w}^{\mu}$, and ${\overline{S_{w}^{\mu}}}^{\sigma}=\overline{\left(S_{\sigma}\right)_{w}^{\mu}}$.

3.2. MV cycles and $\mathbf{M V}$ polytopes. Let $\mu_{1}, \mu_{2}$ be coweights with $\mu_{1} \geq \mu_{2}$. Following Anderson [A], an irreducible component of $\overline{S_{e}^{\mu_{1}} \cap S_{w_{0}}^{\mu_{2}}}$ is called an MV cycle with coweight $\left(\mu_{1}, \mu_{2}\right)$. This definition of an MV cycle is a generalization of the original one in MV]. $X_{*}$ acts on $\mathcal{G}$ by $\nu \cdot L:=t^{\nu} \cdot L$. Since $T$ normalizes $N_{w}$, we see that $\nu \cdot S_{w}^{\mu}=S_{w}^{\mu+\nu}$. If $A$ is a component of $\overline{S_{e}^{\mu_{1}} \cap S_{w_{0}}^{\mu_{2}}}$, then $\nu \cdot A$ is a component of $\overline{S_{e}^{\mu_{1}+\nu} \cap S_{w_{0}}^{\mu_{2}+\nu}}$. Hence $X_{*}$ acts on the set of all MV cycles. The orbit of an MV cycle with coweight $\left(\mu_{1}, \mu_{2}\right)$ is called a stable MV cycle with coweight $\mu_{2}-\mu_{1}$. Note that a stable MV cycle with coweight $\mu$ has a unique representative with coweight $(\nu, \nu+\mu)$ for a fixed coweight $\nu$.

Let $\mathrm{MVC}_{G}$ denote the set of stable MV cycles for $G$, and let $\mathrm{MVC}_{G}^{\mu}$ denote the set of those with coweight $\mu$. For a $T$-invariant closed subvariety $A$ of the affine Grassmannian, let $\Phi(A) \subset t_{\mathbb{R}}:=X_{*} \otimes \mathbb{R}$ be the moment polytope of $A$, which is exactly the convex hull of $\left\{\mu \in X_{*} \mid t^{\mu} \in A\right\}$.

If $A$ is an MV cycle with coweight $\left(\mu_{1}, \mu_{2}\right)$, then we say that $\Phi(A)$ is an MV polytope with coweight $\left(\mu_{1}, \mu_{2}\right)$. The action of $X_{*}$ on the set of MV cycles gives an action of $X_{*}$ on the set of MV polytopes. It is easy to see that $\nu \cdot P=P+\nu$. The orbit of $X_{*}$ on an MV polytope with coweight $\left(\mu_{1}, \mu_{2}\right)$ is called a stable MV polytope with coweight $\mu_{2}-\mu_{1}$. 
Let $\mathrm{MVP}_{G}$ be the set of stable MV polytopes for $G$, and let $\mathrm{MVP}_{G}^{\mu}$ be the set of stable MV polytopes for $G$ with coweight $\mu$. As mentioned in $\mathrm{A}$, there is a natural bijection between $\mathrm{MVC}_{G}$ and $\mathrm{MVP}_{G}$. Let $C$ be an MV cycle, and let $[C]$ be its stable MV cycle. Let $P_{C}$ be the corresponding MV polytope of $C$, and let $\left[P_{C}\right]$ be its stable MV polytope. If there is no confusion, we write $C$ (resp. $P$ ) for both MV cycle (or polytope) and stable MV cycle (resp. polytope).

Suppose we are given a collection of coweights $\mu_{\bullet}=\left(\mu_{w}\right)_{w \in W}$ such that $\mu_{v} \leq_{w}$ $\mu_{w}$ for all $v, w \in W$. Then we define the corresponding pseudo-Weyl polytope by

$$
P\left(\mu_{\bullet}\right):=\bigcap_{w} C_{w}^{\mu_{w}}=\left\{\alpha \mid\left\langle\alpha, w \cdot \lambda_{i}\right\rangle \leq\left\langle\mu_{w}, w \cdot \lambda\right\rangle, \forall w \in W, \text { and } i \in I\right\} .
$$

For a collection $\left(\mu_{w}\right)_{w \in W}$ with coweights such that $\mu_{y} \leq{ }_{w} \mu_{w}$, for any $y, w \in W$, set $A\left(\mu_{\bullet}\right)=\bigcap S_{w}^{\mu_{w}}$, and let $\operatorname{Conv}\left(\mu_{\bullet}\right)$ be the convex hull of $\left(\mu_{w}\right)_{w \in W}$ in $t_{\mathbb{R}} \cdot A\left(\mu_{\bullet}\right)$ is called a GGMS stratum, and it is a candidate of MV cycles. If it is not empty, then the moment polytope of $\overline{A\left(\mu_{\bullet}\right)}$ is exactly $\operatorname{Conv}\left(\mu_{\bullet}\right)$ (see Lemma 2.3, K1]), which also coincides with $P\left(\mu_{\bullet}\right)$. That is, $\operatorname{Conv}\left(\mu_{\bullet}\right)=P\left(\mu_{\bullet}\right)$.

The following theorem gives a criterion for the closure of a GGMS stratum to be an MV cycle.

Theorem 1 (Kamnitzer [K1]). Let $\left(\mu_{w}\right)_{w \in W}$ be the set with coweights, such that $\mu_{y} \leq_{w} \mu_{w}$, for any $y, w \in W$. Then $\overline{A\left(\mu_{\bullet}\right)}=\overline{\bigcap S_{w}^{\mu_{w}}}$ is an $M V$ cycle if and only if $\operatorname{Conv}\left(\mu_{\bullet}\right)$ is an $M V$ polytope.

Let $P$ be an MV polytope with vertices $\left(\mu_{w}\right)_{w \in W}$. Then $P$ is the moment polytope of an MV cycle $\overline{\bigcap S_{w}^{\mu_{w}}}$. In this case, $\sigma\left(\overline{\bigcap S_{w}^{\mu_{w}}}\right)=\overline{\bigcap S_{w}^{\sigma\left(\mu_{\sigma^{-1}(w)}\right)}}$ is also an MV cycle, and its moment polytope is exactly $\operatorname{Conv}\left(\sigma\left(\mu_{\sigma^{-1}(w)}\right)\right)$. Hence it is an MV polytope with vertices $\left(\sigma\left(\mu_{\sigma^{-1}(w)}\right)\right)_{w \in W}$, which coincides with $\sigma(P)$.

Lemma 3.4. Let $\left(\mu_{w}\right)_{w \in W}$ be the vertices of an $M V$ polytope $P$, and let $A\left(\mu_{\bullet}\right)$ be the corresponding GGMS stratum, such that $\overline{A\left(\mu_{\bullet}\right)}$ is an $M V$ cycle. Then the following statements are equivalent:

(1) $P$ is $\sigma$-invariant.

(2) $\overline{A\left(\mu_{\bullet}\right)}$ is $\sigma$-invariant.

(3) $A\left(\mu_{\bullet}\right)$ is $\sigma$-invariant.

(4) $\sigma\left(\mu_{w}\right)=\mu_{\sigma(w)}, \forall w \in W$.

Proof. Since MV cycles are parametrized by MV polytopes bijectively, it is easy to see that the moment polytope of $\sigma\left(\overline{\cap S_{w}^{\mu_{w}}}\right)$ is $\sigma(P)$. So $P$ is $\sigma$-invariant if and only if $\overline{A\left(\mu_{\bullet}\right)}$ is $\sigma$-invariant, i.e., (1) $\Leftrightarrow(2)$.

Assume $\overline{A\left(\mu_{\bullet}\right)}$ is $\sigma$-invariant. Then $\overline{\bigcap S_{w}^{\mu_{w}}}=\overline{\left.\bigcap S_{w}^{\sigma\left(\mu_{\sigma-1}(w)\right.}\right)}$. Since $\bigcap S_{w}^{\mu_{w}}$ and $\bigcap S_{w}^{\sigma\left(\mu_{\sigma^{-1}(w)}\right)}$ are locally closed, we have $\left(\bigcap S_{w}^{\mu_{w}}\right) \cap\left(\bigcap S_{w}^{\sigma\left(\mu_{\sigma-1}(w)\right.}\right) \neq \varnothing$. It implies that, $\forall w \in W, S_{w}^{\mu_{w}} \cap S_{w}^{\sigma\left(\mu_{\sigma^{-1}(w)}\right)} \neq \varnothing$. Hence $\mu_{w}=\sigma\left(\mu_{\sigma^{-1}(w)}\right), \forall w \in W$. So $(2) \Rightarrow(4)$.

It is easy to see (3) $\Leftrightarrow(4)$, and (4) implies (1) immediately.

3.3. Lusztig datum. Let $\mathbf{i}$ be a reduced word, and $n_{\bullet} \in \mathbb{N}^{m}$. Recall some results in [K1. We define $\left\{\mu_{w_{k}^{\mathrm{i}}}\right\}_{0 \leq k \leq m}$ inductively by $\mu_{e}=0$ and $\mu_{w_{k}^{\mathrm{i}}}=\mu_{w_{k-1}^{\mathrm{i}}}-n_{k} w_{k-1}^{\mathrm{i}}\left(\alpha_{i_{k}}^{\vee}\right)$, for any $1 \leq k \leq m$. Set $A^{\mathbf{i}}\left(n_{\bullet}\right)=\bigcap S_{w_{k}^{\mathrm{i}}}^{\mu_{k}^{\mathrm{i}}}$. Then $\overline{A^{\mathrm{i}}\left(n_{\bullet}\right)}$ is an MV cycle with coweight $\mu_{w_{0}}$, and the corresponding MV polytope $P$ has i-Lusztig datum $n_{\bullet}$. 
From the corresponding i-Lusztig datum of the MV polytope $P$, we can recover the vertices of $P$ uniquely, through the above procedure. In this way, we have a bijection from MV polytopes to i-Lusztig data. Moreover, there exists an explicit bijection between i-Lusztig data and MV cycles, $\tau_{\mathbf{i}}: \mathbb{N}^{m} \rightarrow$ MVC by $\tau_{\mathbf{i}}\left(n_{\bullet}\right)=\overline{A^{\mathbf{i}}\left(n_{\bullet}\right)}$.

Let $\mathbf{i}, \mathbf{i}^{\prime}$ be two reduced words of $w_{0}$. It is known that $\mathbf{i}^{\prime}$ can be obtained from $\mathbf{i}$ through several braid moves. Fix a path of braid moves from $\mathbf{i}$ to $\mathbf{i}^{\prime}$. For each move, there is a transform (in Proposition 5.2, K1]) between the Lusztig data of $P$ along the two consecutive reduced words. By combining these transforms, we get a bijection $R_{\mathbf{i}}^{\mathbf{i}^{\prime}}: \mathbb{N}^{m} \rightarrow \mathbb{N}^{m}$, which is independent of the choice of the path from $\mathbf{i}$ to $\mathbf{i}^{\prime}$. We call it the Lusztig transform from $\mathbf{i}$ to $\mathbf{i}^{\prime}$ for $G$. From [K1, we also know that $R_{\mathbf{i}}^{\mathbf{i}^{\prime}}\left(n_{\bullet}\right)=n_{\bullet}^{\prime}$ if and only if $A^{\mathbf{i}}\left(n_{\bullet}\right) \cap A^{\mathbf{i}^{\prime}}\left(n_{\bullet}^{\prime}\right)$ is dense in $A^{\mathbf{i}}\left(n_{\bullet}\right)$.

We give a necessary and sufficient condition on the $\mathbf{i}$-Lusztig datum $n_{\bullet}$, so that $P$ is $\sigma$-invariant. We call such an $n_{\bullet}$ a $\sigma$-invariant i-Lusztig datum.

Proposition 3.5. Let $w_{0}=s_{\eta_{1}} s_{\eta_{2}} \cdots s_{\eta_{m}}$ be a reduced expression of $w_{0}$ relative to the Coxeter group $W^{\sigma}$, where $\eta_{1}, \eta_{2}, \cdots, \eta_{m}$, are orbits of $\sigma$ in $I$. For each $\eta$, we fix a reduced expression of $s_{\eta}$ as an element of $W$, and denote by $\mathbf{i}$ the resulting reduced expression of $w_{0}$ relative to $W$. Let $n_{\bullet}$ be the $\mathbf{i}$-Lusztig datum of $P$. Then $P$ is $\sigma$-invariant if and only if $n_{1}=n_{2}=\cdots=n_{r_{\eta_{1}}}, n_{r_{\eta_{1}}+1}=n_{r_{\eta_{1}}+2}=\cdots=$ $n_{r_{\eta_{1}}+r_{\eta_{2}}}, \cdots$, where $r_{\eta}$ is the length of $s_{\eta}$ as an element of $W$.

Proof. For any orbit $\eta$ of $\sigma$, let $R_{\eta}$ be the root system generated by $\left\{\alpha_{i} ; i \in \eta\right\}$. Let $W_{\eta}$ be the Coxeter group generated by $\left\{s_{i}\right.$, for $\left.i \in \eta\right\}$. Then $s_{\eta}$ is the longest element in $W_{\eta}$.

Recall that $n_{k}$ means the length of the edge connecting $\mu_{w_{k-1}^{\mathrm{i}}}$ with $\mu_{w_{k}^{\mathrm{i}}}$, i.e., $\mu_{w_{k}^{\mathrm{i}}}-\mu_{w_{k-1}^{\mathrm{i}}}=-n_{k} \cdot w_{k-1}^{\mathrm{i}}\left(\alpha_{i_{k}}^{\vee}\right)$. The convex hull of $\left\{\mu_{w} \mid w \in W_{\eta_{1}}\right\}$ forms an $\mathrm{MV}$ polytope for an algebraic group of type $R_{\eta_{1}}$. We denote it by $P_{\eta_{1}}^{1}$. From $\mu_{w_{0}^{\mathbf{i}}}, \cdots, \mu_{w_{r_{1}}^{\mathbf{i}}}$, we get a Lusztig datum $\left(n_{1}, n_{2}, \cdots, n_{r_{\eta_{1}}}\right)$ along the chosen reduced word of $s_{\eta}$. The convex hull of $\left\{\mu_{w} \mid w=s_{\eta_{1}} y\right.$, for $\left.y \in W_{\eta_{2}}\right\}$ forms an MV polytope of type $R_{\eta_{2}}$. We denote it by $P_{\eta_{2}}^{2}$. From $\mu_{w_{r_{\eta_{1}}+1}^{\mathrm{i}}}, \cdots, \mu_{w_{r_{\eta_{1}}+r_{\eta_{2}}}^{\mathrm{i}}}$, we get a Lusztig datum $\left(n_{r_{\eta_{1}}+1}, n_{r_{\eta_{1}}+2}, \cdots, n_{r_{\eta_{1}}+r_{\eta_{2}}}\right)$ along the chosen reduced word of $s_{\eta_{2}}$. Similarly, we get subsequently MV polytopes $P_{\eta_{3}}^{3}, \cdots, P_{\eta_{m}}^{m}$, with type $R_{\eta_{3}}, \cdots, R_{\eta_{m}}$. We also get their corresponding Lusztig data along the chosen reduced words of $s_{\eta_{i}}$.

Now let us return to the proof. If $P$ is $\sigma$-invariant, we have $\sigma\left(\mu_{w}\right)=\mu_{\sigma(w)}$, for all $w \in W$, by Lemma 3.4. Applying Lemma 3.4 again, we see that $P_{\eta_{k}}^{k}$, for all $k$, are $\sigma$-invariant.

Note that there are two possibilities: $A_{2}$ and $A_{1} \times A_{1} \times \cdots \times A_{1}$ ( with $l$ copies of $A_{1}$, where $\ell=2$ or 3 ) for $R_{\eta}$. Hence the sufficient part is reduced to the following two cases which are easy to check.

(1) $A_{2}$, if $P$ is $\sigma$-invariant, then $n_{1}=n_{2}=n_{3}$.

(2) $A_{1} \times A_{1} \times \cdots \times A_{1}$, if $P$ is $\sigma$-invariant, then $n_{1}=n_{2}=\cdots=n_{l}$.

Conversely, from $A^{\mathbf{i}}\left(n_{\bullet}\right)=\bigcap_{k} S_{w_{k}^{\mathrm{i}}}^{\mu_{w_{k}^{\mathrm{i}}}}$, we have $\sigma\left(A^{\mathbf{i}}\left(n_{\bullet}\right)\right)=A^{\mathbf{j}}\left(n_{\bullet}\right)$, where $\mathbf{j}=$ $\left(\sigma\left(i_{1}\right), \sigma\left(i_{2}\right), \cdots, \sigma\left(i_{m}\right)\right)$. From the condition of $n_{\bullet}$, it is easy to see $R_{\mathbf{i}}^{\mathbf{j}}\left(n_{\bullet}\right)=n_{\bullet}$. Hence their closures coincide, i.e., the corresponding MV cycle of this i-Lusztig datum is $\sigma$-invariant. By Lemma 3.4, $P$ is $\sigma$-invariant. 
3.4. The bijection between MV cycles (polytopes) for a symmetric pair. Let $P$ be a $\sigma$-invariant MV polytope for $G$. In this subsection, we will show that $P^{\sigma}$ is an MV polytope for $G^{\sigma}$, and then we get the bijection between MV polytopes for a symmetric pair.

Consider the symmetric pair $\left(A_{4}, B_{2}\right)$. For the longest element in the Weyl group $W$, we have reduced expressions $w_{0}=s_{1} s_{4} \cdot s_{2} s_{3} s_{2} \cdot s_{1} s_{4} \cdot s_{2} s_{3} s_{2}=s_{2} s_{3} s_{2} \cdot s_{1} s_{4} \cdot s_{2} s_{3} s_{2}$. $s_{1} s_{4}$. We get two reduced words $\mathbf{i}_{\sigma}$ and $\mathbf{i}_{\sigma}^{\prime}$ for $G^{\sigma}$ from these two expressions of $w_{0}$. From $\mathbf{i}_{\sigma}$, and $\mathbf{i}_{\sigma}^{\prime}$, we naturally get 2 reduced words for $G, \mathbf{i}=(1,4,2,3,2,1,4,2,3,2)$, $\mathbf{i}^{\prime}=(2,3,2,1,4,2,3,2,1,4)$, respectively. Let $n_{\bullet}, n_{\bullet}^{\prime}$ be Lusztig data along $\mathbf{i}$, and $\mathbf{i}^{\prime}$ for $P$, respectively. According to Proposition 3.5, we may write $n_{\bullet}$ and $n_{\bullet}^{\prime}$ as

$$
\begin{aligned}
& n_{\bullet}=\left(\bar{n}_{1}, \bar{n}_{1}, \bar{n}_{2}, \bar{n}_{2}, \bar{n}_{2}, \bar{n}_{3}, \bar{n}_{3}, \bar{n}_{4}, \bar{n}_{4}, \bar{n}_{4}\right) \in \mathbb{N}^{10}, \\
& n_{\bullet}^{\prime}=\left(\bar{n}_{1}^{\prime}, \bar{n}_{1}^{\prime}, \bar{n}_{1}^{\prime}, \bar{n}_{2}^{\prime}, \bar{n}_{2}^{\prime}, \bar{n}_{3}^{\prime}, \bar{n}_{3}^{\prime}, \bar{n}_{3}^{\prime}, \bar{n}_{4}^{\prime}, \bar{n}_{4}^{\prime}\right) \in \mathbb{N}^{10},
\end{aligned}
$$

where $\bar{n}_{k}, \bar{n}_{k}^{\prime}$ are nonnegative integers.

Set $n_{\bullet}^{\sigma}=\left(\bar{n}_{1}, \bar{n}_{2}, \bar{n}_{3}, \bar{n}_{4}\right)$. By sending $n_{\bullet}$ to $n_{\bullet}^{\sigma}$, we get a bijection between i-Lusztig data of $\sigma$-invariant MV polytopes for $G$ and $\mathbf{i}_{\sigma}$-Lusztig data of MV polytopes for $G^{\sigma}$. We shall show this bijection is intrinsic, and independent of the choice of reduced words. Note that the above procedure works for the general case.

For any subvariety $Y \subset \mathcal{G}$, we set $Y^{\sigma}:=\{y \in Y \mid \sigma(y)=y\}$.

Let $B\left(n_{\bullet}\right)=\left\{\left(b_{\bullet}\right) \in \mathcal{K}^{\ell\left(w_{0}\right)} \mid \operatorname{val}\left(b_{k}\right)=n_{k}, \forall k\right\}$ and $B_{\sigma}\left(n_{\bullet}^{\sigma}\right)=\left\{\left(b_{\bullet}\right) \in \mathcal{K}^{\ell_{\sigma}\left(w_{0}\right)} \mid\right.$ $\left.\operatorname{val}\left(b_{k}\right)=\bar{n}_{k}, \forall k\right\}$, where val is the valuation function on $\mathcal{K}$. Define a map $j_{\sigma}$ from $B_{\sigma}\left(n_{\bullet}^{\sigma}\right)$ to $B\left(n_{\bullet}\right)$, by $j_{\sigma}\left(b_{1}, b_{2}, b_{3}, b_{4}\right)=\left(b_{1}, b_{1}, b_{2}, 2 b_{2}, b_{2}, b_{3}, b_{3}, b_{4}, 2 b_{4}, b_{4}\right)$.

In this subsection, we always assume that $\mathbf{i}$ and $\mathbf{i}^{\prime}$ are reduced words of $G$ resulting from the reduced words of $G^{\sigma}, \mathbf{i}_{\sigma}$ and $\mathbf{i}^{\prime}{ }_{\sigma}$, respectively, in the sense of Proposition 3.5 .

Lemma 3.6. Let $n_{\bullet}$ be a $\sigma$-invariant $\mathbf{i}$-Lusztig datum. Then $A^{\mathbf{i}}\left(n_{\bullet}\right)^{\sigma}=A^{\mathbf{i}_{\sigma}}\left(n_{\bullet}^{\sigma}\right)$.

Proof. We only show this lemma for the pair $\left(A_{4}, B_{2}\right)$, and the following argument works in general.

Let $\iota: A^{\mathbf{i}_{\sigma}}\left(n_{\bullet}^{\sigma}\right) \hookrightarrow \mathcal{G}$ be the natural imbedding, which is the restriction of $\iota: \mathcal{G}_{\sigma} \hookrightarrow \mathcal{G}$. We have surjections $\pi_{\mathbf{i}_{\sigma}}: B_{\sigma}\left(n_{\bullet}^{\sigma}\right) \rightarrow A^{\mathbf{i}_{\sigma}}\left(n_{\bullet}^{\sigma}\right)$, and $\pi_{\mathbf{i}}: B\left(n_{\bullet}\right) \rightarrow A^{\mathbf{i}}\left(n_{\bullet}\right)$, which are given by

$$
\begin{aligned}
\pi_{\mathbf{i}_{\sigma}}\left(b_{1}, b_{2}, b_{3}, b_{4}\right)= & {\left[\eta_{w_{0}}^{-1}\left(x_{\eta_{1}}\left(b_{1}\right) x_{\eta_{2}}\left(b_{2}\right) x_{\eta_{1}}\left(b_{3}\right) x_{\eta_{2}}\left(b_{4}\right)\right)\right], } \\
& \pi_{\mathbf{i}}\left(b_{1}, b_{1}, b_{2}, 2 b_{2}, b_{2}, b_{3}, b_{3}, b_{4}, 2 b_{4}, b_{4}\right) \\
= & {\left[\eta _ { w _ { 0 } } ^ { - 1 } \left(x_{1}\left(b_{1}\right) x_{4}\left(b_{1}\right) \cdot x_{2}\left(b_{2}\right) x_{3}\left(2 b_{2}\right) x_{2}\left(b_{2}\right) \cdot x_{1}\left(b_{3}\right) x_{4}\left(b_{3}\right)\right.\right.} \\
& \left.\left.\cdot x_{2}\left(b_{4}\right) x_{3}\left(2 b_{4}\right) x_{2}\left(b_{4}\right)\right)\right],
\end{aligned}
$$

where $x_{\eta_{1}}$ and $x_{\eta_{2}}$ are root subgroup homomorphisms for $G^{\sigma}$, and we denote by [] the projection from $G(\mathcal{K})$ to $\mathcal{G}$. For the definition of $\eta_{w_{0}}$, see section 4.4, [K1]. Since $x_{1}\left(b_{i}\right) x_{4}\left(b_{i}\right)=x_{\eta_{1}}\left(b_{i}\right)$, for $\mathrm{i}=1$ or 3 , and $x_{2}\left(b_{j}\right) x_{3}\left(2 b_{j}\right) x_{2}\left(b_{j}\right)=x_{\eta_{2}}\left(b_{j}\right)$, for $\mathrm{j}=2$ or 4 , we can see that $\iota \circ \pi_{\mathbf{i}_{\sigma}}=\pi_{\mathbf{i}} \circ j_{\sigma}$, i.e., we have the following commutative diagram:

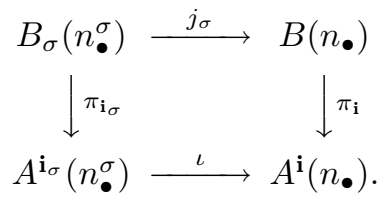

Since $\pi_{\mathbf{i}_{\sigma}}\left(B_{\sigma}\left(n_{\bullet}^{\sigma}\right)\right)=A^{\mathbf{i}_{\sigma}}\left(n_{\bullet}^{\sigma}\right)$, we have $A^{\mathbf{i}_{\sigma}}\left(n_{\bullet}^{\sigma}\right) \subset A^{\mathbf{i}}\left(n_{\bullet}\right)^{\sigma}$. 
Assume $n_{\bullet}$ is of coweight $\mu$. It is known that $X(\mu)=S_{e}^{0} \cap S_{w_{0}}^{\mu}=\bigsqcup A^{\mathbf{i}}\left(n_{\bullet}^{\prime}\right)$, where the union is taken over $n_{\bullet}^{\prime}$, such that $n_{\bullet}^{\prime}$ is an $\mathbf{i}^{\prime}$-Lusztig datum with coweight $\mu$. Hence we have

$$
X(\mu)^{\sigma}=\bigsqcup A^{\mathbf{i}}\left(n_{\bullet}^{\prime}\right)^{\sigma}
$$

where $A^{\mathbf{i}}\left(n_{\bullet}\right)$ appear in the right-hand side.

From Corollary 3.3, we have the decomposition

$$
X(\mu)^{\sigma}=\bigsqcup A^{\mathbf{i}_{\sigma}}\left(m_{\bullet}\right),
$$

where the union is taken over $m_{\bullet}$ such that $m_{\bullet}$ is an $\mathbf{i}_{\sigma}$-Lusztig datum with coweight $\mu$.

Let $m_{\bullet}=\left(m_{1}, m_{2}, m_{3}, m_{4}\right)$ be an $\mathbf{i}_{\sigma}$-Lusztig datum, such that $\overline{A^{\mathbf{i}_{\sigma}}\left(m_{\bullet}\right)}$ is an MV cycle for $G^{\sigma}$ with coweight $\mu$. Let $n_{\bullet}^{\prime \prime}=\left(m_{1}, m_{1}, m_{2}, m_{2}, m_{2}, m_{3}, m_{3}, m_{4}, m_{4}, m_{4}\right)$. Then $n_{\bullet}^{\prime \prime}$ is $\sigma$-invariant, and hence $A^{\mathbf{i}_{\sigma}}\left(m_{\bullet}\right) \subset A^{\mathbf{i}}\left(n_{\bullet}^{\prime \prime}\right)^{\sigma}$. By comparing decompositions of $X(\mu)^{\sigma}$ in (1) and (2), we obtain $A^{\mathbf{i}}\left(n_{\bullet}\right)^{\sigma}=A^{\mathbf{i}_{\sigma}}\left(n_{\bullet}^{\sigma}\right)$.

Remark 3.1. From this lemma, we see that the closure of the fixed point set of $\sigma$ on some open subset of a $\sigma$-invariant MV cycle $C$ is an MV cycle for $G^{\sigma}$. We believe that the fixed point set of $\sigma$ on a $\sigma$-invariant MV cycle for $G$ is an MV cycle for $G^{\sigma}$.

Corollary 3.7. If $\overline{A^{\mathbf{i}}\left(n_{\bullet}\right)}$ is not $\sigma$-invariant, then $A^{\mathbf{i}}\left(n_{\bullet}\right)^{\sigma}$ is empty.

Lemma 3.8. If $n_{\bullet}$ is a $\sigma$-invariant $\mathbf{i}$-Lusztig datum, and $R_{\mathbf{i}}^{\mathbf{i}^{\prime}}\left(n_{\bullet}\right)=n_{\bullet}^{\prime}$, then $\left(A^{\mathbf{i}}\left(n_{\bullet}\right) \cap A^{\mathbf{i}^{\prime}}\left(n_{\bullet}^{\prime}\right)\right)^{\sigma}$ contains an open dense subset.

Proof. We can change $\mathbf{i}$ to $\mathbf{i}^{\prime}$ by combining several braid $d$-moves.

If $\left(\cdots, i_{k}, i_{k+1}, i_{k+2}, i_{k+3}, \cdots\right) \mapsto\left(\cdots, i_{k}, i_{k+2}, i_{k+1}, i_{k+3}, \cdots\right),(d=2)$, define a rational map from $B\left(n_{\bullet}\right)$ to $B\left(n_{\bullet}^{\prime}\right)$, by

$$
\left(\cdots, b_{k}, b_{k+1}, b_{k+2}, b_{k+3}, \cdots\right) \mapsto\left(\cdots, b_{k}, b_{k+2}, b_{k+1}, b_{k+3}, \cdots\right) .
$$

If $\left(\cdots, i_{k}, i_{k+1}, i_{k+2}, i_{k+3}, i_{k+4}, \cdots\right) \mapsto\left(\cdots, i_{k}, i_{k+2}, i_{k+1}, i_{k+2}, i_{k+4}, \cdots\right)$, $(d=3)$, where $i_{k+1}=i_{k+3}$, then we define a rational map from $B\left(n_{\bullet}\right)$ to $B\left(n_{\bullet}^{\prime}\right)$ by

$$
\begin{aligned}
\left(\cdots, b_{k}, b_{k+1}, b_{k+2}, b_{k+3}, b_{k+4} \cdots\right) & \\
& \mapsto\left(\cdots, b_{k}, \frac{b_{k+2} b_{k+3}}{b_{k+1}+b_{k+3}}, b_{k+1}+b_{k+3}, \frac{b_{k+1} b_{k+2}}{b_{k+1}+b_{k+3}}, b_{k+4}, \cdots\right) .
\end{aligned}
$$

It is well known that, by several braid $d$-moves, we can arrive at $\mathbf{i}^{\prime}$ from $\mathbf{i}$. Let $\mathbf{i} \mapsto \mathbf{i}_{1} \mapsto \mathbf{i}_{2} \mapsto \cdots \mapsto \mathbf{i}^{\prime}$ be one such path, where $\mapsto$ represents a braid $d$-move. For a path from $\mathbf{i}$ to $\mathbf{i}^{\prime}$, we denote the rational map $f$ by combining those in every step defined above. Assume $f\left(b_{1}, \cdots, b_{m}\right)=\left(b_{1}^{\prime}, \cdots, b_{m}^{\prime}\right)$. It is easy to see that $b_{k}^{\prime}$ is a rational function with numerator and denominator as nonzero polynomials with nonnegative integral coefficients. Consider the diagram

$$
\begin{array}{ll}
B\left(n_{\bullet}\right) \rightarrow & B\left(n_{\bullet}^{\prime}\right) \\
\downarrow & \quad \pi_{\mathbf{i}} \quad \downarrow \pi_{\mathbf{i}^{\prime}} \\
A^{\mathbf{i}}\left(n_{\bullet}\right) \rightarrow A^{\mathbf{i}^{\prime}}\left(n_{\bullet}^{\prime}\right)
\end{array}
$$

where $\pi_{\mathbf{i}}$ is as in the proof of Lemma 3.6, and dashed arrows denote rational maps. We have $\pi_{\mathbf{i}}=\pi_{\mathbf{i}^{\prime}} \circ f$. 
Let $F$ be the product of all denominators appearing in every step of $d$-moves, so it is a nonzero polynomial with nonnegative integral coefficients. Let $U=\left\{\left(b_{\bullet}\right) \in\right.$ $\left.B\left(n_{\bullet}\right) \mid F\left(b_{\bullet}\right) \neq 0\right\}$. Then $f$ is well defined on $U$, and so $\pi_{\mathbf{i}}(U) \subset A^{\mathbf{i}}\left(n_{\bullet}\right) \cap A^{\mathbf{i}^{\prime}}\left(n_{\bullet}^{\prime}\right)$.

There exists $y \in U$, such that $\pi_{\mathbf{i}}(y) \in \pi_{\mathbf{i}}(U) \subset A^{\mathbf{i}}\left(n_{\bullet}\right) \cap A^{\mathbf{i}^{\prime}}\left(n_{\bullet}^{\prime}\right)$, and $\pi_{\mathbf{i}}(y)$ is $\sigma$-invariant. Hence $\left(A^{\mathbf{i}}\left(n_{\bullet}\right) \cap A^{\mathbf{i}^{\prime}}\left(n_{\bullet}^{\prime}\right)\right)^{\sigma}$ is nonempty. Since $\pi_{\mathbf{i}}$ is an open map, $\pi_{\mathbf{i}}(U)$ is open in $A^{\mathbf{i}}\left(n_{\bullet}\right)$. We only show it in the case of $\left(A_{4}, B_{2}\right)$. Since $\overline{A^{\mathbf{i}}\left(n_{\bullet}\right)}$ is $\sigma$-invariant, we have $n_{\bullet}=\left(\bar{n}_{1}, \bar{n}_{1}, \bar{n}_{2}, \bar{n}_{2}, \bar{n}_{2}, \bar{n}_{3}, \bar{n}_{3}, \bar{n}_{4}, \bar{n}_{4}, \bar{n}_{4}\right)$. Now take $y=$ $\left(t^{\bar{n}_{1}}, t^{\bar{n}_{1}}, t^{\bar{n}_{2}}, 2 t^{\bar{n}_{2}}, t^{\bar{n}_{2}}, t^{\bar{n}_{3}}, t^{\bar{n}_{3}}, t^{\bar{n}_{4}}, 2 t^{\bar{n}_{4}}, t^{\bar{n}_{4}}\right) \in B\left(n_{\bullet}\right)$, then $F(y) \neq 0$. In the general case, we have a similar argument.

Since $A^{\mathbf{i}}\left(n_{\bullet}\right)$ is irreducible by Lemma 3.6, we have $\left(A^{\mathbf{i}}\left(n_{\bullet}\right) \cap A^{\mathbf{i}^{\prime}}\left(n_{\bullet}^{\prime}\right)\right)^{\sigma}$ is dense in $A^{\mathbf{i}}\left(n_{\bullet}\right)^{\sigma}$.

Lemma 3.9. Let $\operatorname{Conv}\left(\left(\mu_{w}\right)_{w \in W^{\sigma}}\right)$ be the convex hull of $\left(\mu_{w}\right)_{w \in W^{\sigma}}$ in $t_{\mathbb{R}}$. If the $M V$ polytope $P=\operatorname{Conv}\left(\left(\mu_{w}\right)_{w \in W}\right)$ is $\sigma$-invariant, then $P^{\sigma}=\operatorname{Conv}\left(\left(\mu_{w}\right)_{w \in W^{\sigma}}\right)$.

Proof. Since $P$ is $\sigma$-invariant, we have $\sigma\left(\mu_{w}\right)=\mu_{w}$, for $w \in W^{\sigma}$. We can easily see that $\sigma$ acts trivially on $\operatorname{Conv}\left(\left(\mu_{w}\right)_{w \in W^{\sigma}}\right)$, so $\operatorname{Conv}\left(\left(\mu_{w}\right)_{w \in W^{\sigma}}\right) \subset P^{\sigma}$.

For the converse, the perfect pairing $\left(X_{*} \otimes \mathbb{R}\right) \times\left(X^{*} \otimes \mathbb{R}\right) \rightarrow \mathbb{R}$ descends to $\left(X_{*}^{\sigma} \otimes \mathbb{R}\right) \times\left(X_{\sigma}^{*} \otimes \mathbb{R}\right) \rightarrow \mathbb{R}$ (see Section 2.2). Note that $t_{\mathbb{R}}^{\sigma}$ can be identified with $X_{*}^{\sigma} \otimes \mathbb{R}$.

For any $\beta \in P^{\sigma} \subset P$, and $w \in W^{\sigma}$, we have $\left\langle\beta, w \cdot \lambda_{i}\right\rangle \leq\left\langle\mu_{w}, w \cdot \lambda_{i}\right\rangle$. By descent, we have $\left\langle\beta, w \cdot \lambda_{\eta}\right\rangle \leq\left\langle\mu_{w}, w \cdot \lambda_{\eta}\right\rangle$, for every orbit $\eta$ of $\sigma$ in $I$, where $\lambda_{\eta}$ is the fundamental weight for $G^{\sigma}$ corresponding to $\lambda_{i}$, for $i \in I$. Since $P^{\sigma} \subset t_{\mathbb{R}}^{\sigma}$, we see that

$$
P^{\sigma} \subset\left\{\beta \in t_{\mathbb{R}}^{\sigma} \mid\left\langle\beta, w \cdot \lambda_{\eta}\right\rangle \leq\left\langle\mu_{w}, w \cdot \lambda_{\eta}\right\rangle, \forall \eta, \forall w \in W^{\sigma}\right\} .
$$

The right-hand side is exactly $\operatorname{Conv}\left(\left(\mu_{w}\right)_{w \in W^{\sigma}}\right)$.

Theorem 3.10. If $P$ is a $\sigma$-invariant $M V$ polytope for $G$, then $P^{\sigma}$ is an $M V$ polytope for $G^{\sigma}$.

Proof. Let $\mu_{\bullet}$ be the vertices of $P$. Fix a reduced word $\mathbf{i}_{\sigma}$ for $G^{\sigma}$, and let $n_{\bullet}^{\sigma}$ be the corresponding $\mathbf{i}_{\sigma}$-Lusztig datum of $P$.

Let $\mathbf{i}$ be the fixed reduced word for $G$ from $\mathbf{i}_{\sigma}$, in the sense of Proposition 3.5. Let $J=\left\{\left(\mathbf{i}^{\prime}, n_{\bullet}^{\prime}\right) \mid \mathbf{i}^{\prime}\right.$ be a reduced word for $G$ from some reduced word $\mathbf{i}_{\sigma}^{\prime}$ for $G^{\sigma}$, and $\left.R_{\mathbf{i}}^{\mathbf{i}^{\prime}}\left(n_{\bullet}\right)=n_{\bullet}^{\prime}\right\}$. We have $\bigcap_{\left(\mathbf{i}^{\prime}, n_{\bullet}^{\prime}\right) \in J} A^{\mathbf{i}^{\prime}}\left(n_{\bullet}^{\prime}\right)^{\sigma}$ contains an open and dense subset of $A^{\mathbf{i}}\left(n_{\bullet}\right)^{\sigma}$ from Lemma 3.8, since the intersection of finite open dense subsets is still open and dense.

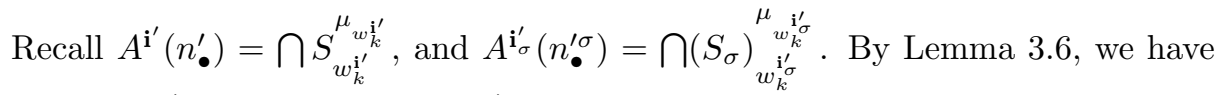
$\left(\bigcap_{\left(\mathbf{i}^{\prime}, n_{\bullet}^{\prime}\right) \in J} A^{\mathbf{i}^{\prime}}\left(n_{\bullet}^{\prime}\right)\right)^{\sigma}=\bigcap_{\left(\mathbf{i}_{\sigma}^{\prime}, n_{\bullet}^{\prime} \sigma\right)} A^{\mathbf{i}_{\sigma}^{\prime}}\left(n_{\bullet}^{\prime \sigma}\right)=A\left(\left(\mu_{w}\right)_{w \in W^{\sigma}}\right)$, where $A\left(\left(\mu_{w}\right)_{w \in W^{\sigma}}\right)=$ $\bigcap_{w \in W^{\sigma}}\left(S_{\sigma}\right)_{w}^{\mu_{w}}$. The last equality holds, since for any $w \in W^{\sigma}$, there exists some reduced word $\mathbf{i}_{\sigma}^{\prime}$ of $G^{\sigma}$ and some integer $k$, such that $w=w_{k}^{\mathbf{i}_{\sigma}^{\prime}}$. Therefore, we have $\overline{A^{\mathbf{i}_{\sigma}\left(n_{\bullet}^{\sigma}\right)}}=\overline{A^{\mathbf{i}}\left(n_{\bullet}\right)^{\sigma}}=\overline{\left(\bigcap_{\left(\mathbf{i}^{\prime}, n_{\bullet}^{\prime}\right) \in J} A^{\mathbf{i}^{\prime}}\left(n_{\bullet}^{\prime}\right)\right)^{\sigma}}=\overline{A\left(\left(\mu_{w}\right)_{w \in W^{\sigma}}\right)}$. That means, the moment polytope of the MV cycle $\overline{A^{\mathbf{i}_{\sigma}\left(n_{\bullet}\right)}}$ is $\operatorname{Conv}\left(\left(\mu_{w}\right)_{w \in W^{\sigma}}\right)$, which is exactly $P^{\sigma}$, by Lemma 3.9. Hence $P^{\sigma}$ is really an MV polytope for $G^{\sigma}$.

Corollary 3.11. Let $\left(\mathbf{i}, n_{\bullet}\right)$ and $\left(\mathbf{i}^{\prime}, n_{\bullet}^{\prime}\right)$ be two $\sigma$-invariant Lusztig data. If $R_{\mathbf{i}}^{\mathbf{i}^{\prime}}\left(n_{\bullet}\right)$ $=n_{\bullet}^{\prime}$, then $R_{\mathbf{i}_{\sigma}}^{\mathbf{i}_{\sigma}^{\prime}}\left(n_{\bullet}^{\sigma}\right)=n_{\bullet}^{\prime \sigma}$ 
Theorem 3.12. We have a bijection $\theta_{P}: \mathrm{MVP}_{G}^{\sigma} \longrightarrow \mathrm{MVP}_{G^{\sigma}}$, given by $P \mapsto P^{\sigma}$, which preserves coweights. Induced from $\theta_{P}$, we have a bijection $\theta_{C}: \mathrm{MVC}_{G}^{\sigma} \longrightarrow$ $\mathrm{MVC}_{G^{\sigma}}$

Proof. Let $P$ be a $\sigma$-invariant MV polytope for $G$. By Theorem 3.10, we have a well-defined map $\theta_{P}: \mathrm{MVP}_{G}^{\sigma} \longrightarrow \mathrm{MVP}_{G^{\sigma}}$ by $\theta_{P}(P)=P^{\sigma}$.

Fix a reduced word $\mathbf{i}_{\sigma}$ for $G^{\sigma}$. Let $\mathbf{i}$ be a reduced word coming from $\mathbf{i}_{\sigma}$. For any MV polytope for $G$ (resp. $G^{\sigma}$ ), we have the corresponding $\mathbf{i}$ (resp. $\mathbf{i}_{\sigma}$ ) Lusztig datum. According to Proposition 3.5, $\theta_{P}$ is injective. Let $Q$ be any MV polytope for $G^{\sigma}$, and let $m_{\bullet}$ be the $\mathbf{i}_{\sigma}$-Lusztig datum of $Q$. By Lemma 3.6 and its proof, there exists a unique $\mathbf{i}$-Lusztig datum $n_{\bullet}$ such that $A^{\mathbf{i}_{\sigma}}\left(m_{\bullet}\right)$ is contained in $A^{\mathbf{i}}\left(n_{\bullet}\right)$, and $n_{\bullet}$ is $\sigma$-invariant. Let $P_{Q}$ be the MV polytope of $\overline{A^{\mathbf{i}}\left(n_{\bullet}\right)}$. We have $P_{Q}^{\sigma}=Q$, since $P_{Q}^{\sigma}$ has the same $\mathbf{i}_{\sigma}$-Lusztig datum as $Q$. So $\theta_{P}$ is surjective.

Hence $\theta_{P}$ is a bijection, and it is easy to see that it preserves the coweights of MV polytopes.

3.5. The bijection in the highest weight case. Let $\lambda, \mu$ be $\sigma$-invariant coweights, we set $X(\lambda, \mu):=S_{e}^{\lambda} \cap S_{w_{0}}^{\mu}$, and $X(\mu-\lambda)=S_{e}^{0} \cap S_{w_{0}}^{\mu-\lambda}$. In this subsection, we have the same assumptions on $\mathbf{i}$ and $\mathbf{i}_{\sigma}$ as in Subsection 3.4 .

The following lemma is given by Anderson [A]

Lemma 3.13. An irreducible component of $X(\lambda, \mu)$ is contained in $\overline{\mathcal{G}^{\lambda}}$ if and only if it appears as basis in $V_{\mu}(\lambda)$

First, we have the decomposition

$$
X(\lambda, \mu)=\lambda \cdot X(\mu-\lambda)=\bigsqcup \lambda \cdot A^{\mathbf{i}}\left(n_{\bullet}\right),
$$

where the union is taken over $n$ • which are $\mathbf{i}$-Lusztig data with coweight $\mu-\lambda$. Then

$$
S_{e}^{\lambda} \cap S_{w_{0}}^{\mu} \cap \overline{\mathcal{G}^{\lambda}}=\bigsqcup_{1} \lambda \cdot A^{\mathrm{i}}\left(n_{\bullet}\right) \cup \bigsqcup_{2}\left(\lambda \cdot A^{\mathrm{i}}\left(n_{\bullet}\right) \cap \overline{\mathcal{G}^{\lambda}}\right),
$$

where the first union 1 is taken over those $n_{\bullet}$ in (3) such that $\lambda \cdot A^{\mathrm{i}}\left(n_{\bullet}\right) \subset \overline{\mathcal{G}^{\lambda}}$; the second union 2 is taken over those $n_{\bullet}$ in (3) such that $\lambda \cdot A^{\mathrm{i}}\left(n_{\bullet}\right) \nsubseteq \overline{\mathcal{G}^{\lambda}}$.

If $\lambda \cdot A^{\mathrm{i}}\left(n_{\bullet}\right) \nsubseteq \overline{\mathcal{G}^{\lambda}}$, then $\lambda \cdot A^{\mathrm{i}}\left(n_{\bullet}\right) \cap \overline{\mathcal{G}^{\lambda}}$ is of lower dimension than $A^{\mathrm{i}}\left(n_{\bullet}\right)$.

From decomposition (4) and Corollary 3.7, we have

(5) $\left(S_{e}^{\lambda} \cap S_{w_{0}}^{\mu} \cap \overline{\mathcal{G}^{\lambda}}\right)^{\sigma}=\left(S_{e}^{\lambda}\right)^{\sigma} \cap\left(S_{w_{0}}^{\mu}\right)^{\sigma} \cap\left(\overline{\mathcal{G}^{\lambda}}\right)^{\sigma}=\bigsqcup_{3} \lambda \cdot A^{\mathrm{i}}(n \bullet)^{\sigma} \cup \bigsqcup_{4}\left(\lambda \cdot A^{\mathbf{i}}(n \bullet) \cap \overline{\mathcal{G}^{\lambda}}\right)^{\sigma}$,

where the first union 3 is taken over those $n_{\bullet}$ in (3), such that $\lambda \cdot A^{\mathbf{i}}\left(n_{\bullet}\right) \subset \overline{\mathcal{G}^{\lambda}}$ and $n_{\bullet}$ is $\sigma$-invariant; the second union 4 is taken over those $n_{\bullet}$ in (3), such that $\lambda \cdot A^{\mathbf{i}}\left(n_{\bullet}\right) \nsubseteq \overline{\mathcal{G}^{\lambda}}$ and $n_{\bullet}$ is $\sigma$-invariant. From the viewpoint of $G^{\sigma}$, we also have the decomposition

$$
\left(S_{\sigma}\right)_{e}^{\lambda} \cap\left(S_{\sigma}\right)_{w_{0}}^{\mu} \cap\left(\overline{\mathcal{G}^{\lambda}}\right)^{\sigma}=\bigsqcup_{5} \lambda \cdot A^{\mathbf{i}_{\sigma}}\left(m_{\bullet}\right) \cup \bigsqcup_{6}\left(\lambda \cdot A^{\mathbf{i}_{\sigma}}\left(m_{\bullet}\right) \cap \overline{\mathcal{G}_{\sigma}^{\lambda}}\right),
$$

where the first union 5 is taken over $m_{\bullet}$ which are $\mathbf{i}_{\sigma}$-Lusztig data with coweight $\mu-\lambda$, satisfying $\lambda \cdot A^{\mathbf{i}_{\sigma}}\left(m_{\bullet}\right) \subset \overline{\mathcal{G}_{\sigma}^{\lambda}}$; the second union 6 is taken over $m_{\bullet}$ which are $\mathbf{i}_{\sigma}$-Lusztig data with coweight $\mu-\lambda$, satisfying $\lambda \cdot A^{\mathbf{i}_{\sigma}}\left(m_{\bullet}\right) \nsubseteq \overline{\mathcal{G}_{\sigma}^{\lambda}}$.

If $\lambda \cdot A^{\mathbf{i}_{\sigma}}\left(m_{\bullet}\right) \not \subseteq \overline{\mathcal{G}^{\lambda}}$, then $\lambda \cdot A^{\mathbf{i}_{\sigma}}\left(m_{\bullet}\right) \cap \overline{\mathcal{G}_{\sigma}^{\lambda}}$ is of lower dimension than $A^{\mathbf{i}_{\sigma}}\left(m_{\bullet}\right)$. 
Lemma 3.14. $\overline{\mathcal{G}^{\lambda}}=\overline{\bigcap S_{w}^{w \cdot \lambda}}$.

Proof. We know that $\overline{\bigcap S_{w}^{w \cdot \lambda}}$ is an MV cycle with coweight $\left(\lambda, w_{0} \cdot \lambda\right)$, and it is contained in $\overline{\mathcal{G}^{\lambda}}$. Since both of them are of the same dimension $2\langle\lambda, \rho\rangle$, and both of them are irreducible, we have $\overline{\mathcal{G}^{\lambda}}=\overline{\bigcap S_{w}^{w . \lambda}}$.

Lemma 3.15. If $\lambda \cdot A^{\mathbf{i}}\left(n_{\bullet}\right) \nsubseteq \overline{\mathcal{G}^{\lambda}}$, and $n_{\bullet}$ is $\sigma$-invariant, then $\left(\lambda \cdot A^{\mathbf{i}}\left(n_{\bullet}\right) \cap \overline{\mathcal{G}^{\lambda}}\right)^{\sigma}$ is of lower dimension than $A^{\mathrm{i}}\left(n_{\bullet}\right)^{\sigma}$.

Proof. With the same reason as in the proof of Lemma 3.6. we can find an open subset $U \subset B\left(n_{\bullet}\right)$, such that $\pi_{\mathbf{i}}(U) \subset \bigcap_{\left(\mathbf{i}, n_{\bullet}\right)} A^{\mathbf{i}}\left(n_{\bullet}\right)=\bigcap_{w} S_{w}^{\mu_{w}}$ is open in $A^{\mathbf{i}}\left(n_{\bullet}\right)$.

Note that $\left(\cap \lambda \cdot S_{w}^{\mu_{w}}\right) \cap \overline{\mathcal{G}^{\lambda}}$ is empty. Otherwise, if there exists a point $p \in$ $\left(\cap \lambda \cdot S_{w}^{\mu_{w}}\right) \cap \overline{\mathcal{G}^{\lambda}}$, then

$$
p \in\left(\bigcap \lambda \cdot S_{w}^{\mu_{w}}\right) \cap \overline{\mathcal{G}^{\lambda}}=\left(\bigcap \lambda \cdot S_{w}^{\mu_{w}}\right) \cap \overline{\cap S_{w}^{w \cdot \lambda}} \subset\left(\bigcap \lambda \cdot S_{w}^{\mu_{w}}\right) \cap \overline{S_{w}^{w \cdot \lambda}} .
$$

That is, $\forall w \in W, p$ must be contained in $\lambda \cdot S_{w}^{\mu_{w}} \cap \overline{S_{w}^{w \cdot \lambda}}$. From $\overline{S_{w}^{w \cdot \lambda}}=\bigsqcup_{\mu<_{w} \cdot \lambda} S_{w}^{\mu}$, we have $\mu_{w}+\lambda \leq_{w} w \cdot \lambda$. We get that $\operatorname{Conv}\left(\mu_{\bullet}\right)+\lambda \subset \operatorname{Conv}(W \cdot \lambda)$. According to Anderson's theorem on multiplicity of weight space $\left[\mathrm{A}\right.$, we have $\lambda \cdot \overline{A\left(\mu_{\bullet}\right)}$ is an MV cycle in $V_{\mu}(\lambda)$. By Lemma 3.13, it is a contradiction to the condition that $\lambda \cdot A^{\mathbf{i}}\left(n_{\bullet}\right) \nsubseteq \overline{\mathcal{G}^{\lambda}}$. As in Lemma 3.8, there exists a point $p \in \lambda \cdot A^{\mathbf{i}}\left(n_{\bullet}\right)$. So $\lambda \cdot A^{\mathbf{i}}\left(n_{\bullet}\right)^{\sigma} \cap{\overline{\mathcal{G}^{\lambda}}}^{\sigma}$ has lower dimension than $A^{\mathbf{i}}\left(n_{\bullet}\right)^{\sigma}$.

By Lemma 3.15, and by comparing the two decompositions (5) and (6), we have that the set $\left\{A^{\mathbf{i}}\left(n_{\bullet}\right) \mid n_{\bullet}\right.$ is $\sigma$-invariant and is of coweight $\mu-\lambda$, and $\left.\lambda \cdot A^{\mathbf{i}}\left(n_{\bullet}\right) \subseteq \overline{\mathcal{G}^{\lambda}}\right\}$ is in bijection with the set $\left\{A^{\mathbf{i}_{\sigma}}\left(m_{\bullet}\right) \mid m_{\bullet}\right.$ is of coweight $\mu-\lambda$, and $\left.\lambda \cdot A^{\mathbf{i}_{\sigma}}\left(m_{\bullet}\right) \subseteq \overline{\mathcal{G}_{\sigma}^{\lambda}}\right\}$, by sending $A^{\mathrm{i}}\left(n_{\bullet}\right)$ to $A^{\mathrm{i}}\left(n_{\bullet}\right)^{\sigma}$. We thus obtain the following theorem.

Theorem 3.16. We have a bijection $\theta_{C}^{\lambda}: \operatorname{MVC}_{G}(\lambda)^{\sigma} \longrightarrow \operatorname{MVC}_{G_{\sigma}}(\lambda)$, which is the restriction of $\theta_{C}$ in Theorem 3.12 .

\section{TWining CHARACTER FORMUla}

Recall that $\operatorname{Perv}_{G(\mathcal{O})}(\mathcal{G})$ is a tensor category [MV], and it is easy to see the tensor functor $\sigma^{*}$ induced from the action of $\sigma$ on affine Grassmannian is a tensor equivalence. From the functoriality of Tannakian formalism [DM], we have a natural automorphism $\bar{\sigma}$ on $G^{\vee}$.

Fix a $\sigma$-invariant coweight $\lambda$, and choose an isomorphism $\phi: I C_{\lambda} \simeq \sigma^{*}\left(I C_{\lambda}\right)$, which is compatible with the action of $\sigma$ on MV cycles (as the basis of $V(\lambda)$ ).

Lemma 4.1. The action of $\bar{\sigma}$ on $G^{\vee}$ is compatible with the natural action of $\sigma$ on $V(\lambda)$ induced from $\phi$.

Proof. Let $T$ be the functor from $\operatorname{Perv}_{G(\mathcal{O})}(\mathcal{G})$ to $\operatorname{Rep}\left(G^{\vee}\right)$, such that $T\left(I C_{\lambda}\right)=$ $\left(\rho_{\lambda}, V(\lambda)\right)$, where $\rho_{\lambda}: G^{\vee} \rightarrow G L(V(\lambda))$ is the corresponding representation.

From $\sigma^{*}: \operatorname{Perv}_{G(\mathcal{O})}(\mathcal{G}) \rightarrow \operatorname{Perv}_{G(\mathcal{O})}(\mathcal{G})$, we get $T\left(\sigma^{*}\left(I C_{\lambda}\right)\right)=\left(\rho_{\lambda} \circ \bar{\sigma}, V(\lambda)\right)$. Let $\tilde{\sigma}$ be the functor from $\operatorname{Rep}\left(G^{\vee}\right)$ to $\operatorname{Rep}\left(G^{\vee}\right)$, by sending $\left(\rho_{\lambda}, V(\lambda)\right)$ to $\left(\rho_{\lambda} \circ \bar{\sigma}, V(\lambda)\right)$. Then we have the following commutative diagram:

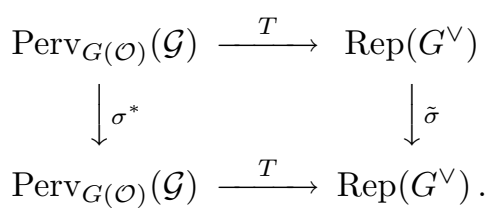


By applying $T$ to $\phi: I C_{\lambda} \simeq \sigma^{*}\left(I C_{\lambda}\right)$, we obtain an isomorphism $\sigma=T(\phi)$ : $\left(\rho_{\lambda}, V(\lambda)\right) \rightarrow\left(\rho_{\lambda} \circ \bar{\sigma}, V(\lambda)\right)$ in $\operatorname{Rep}\left(G^{\vee}\right)$. In other words, there exists a linear isomorphism $\sigma: V(\lambda) \rightarrow V(\lambda)$ satisfying

$$
\sigma\left(\rho_{\lambda}(g) \cdot v\right)=\left(\rho_{\lambda} \circ \bar{\sigma}\right)(g) \cdot \sigma(v)=\rho_{\lambda}(\bar{\sigma}(g)) \cdot \sigma(v),\left(g \in G^{\vee}, v \in V(\lambda)\right) .
$$

Theorem 4.2. $\bar{\sigma}$ is a Dynkin automorphism on $G^{\vee}$.

Proof. Let Vect $X_{*}$ be the tensor category of $X_{*}$-graded vector spaces. The action of $\sigma$ on $X_{*}$ induces an tensor functor $\sigma^{\circ}$ on Vect $X_{*}$. From Mirkovic-Vilonen's paper [MV], we know that there is a tensor functor $F$ from $\operatorname{Perv}_{G(\mathcal{O})}(\mathcal{G})$ to $\operatorname{Vect}_{X_{*}}$, and it is easy to see $\sigma^{*}$ and $\sigma^{\circ}$ are compatible with $F$.

Applying Tannkian formalism, from $F$ we get the forgetful functor from $\operatorname{Rep}\left(G^{\vee}\right)$ to $\operatorname{Rep}\left(T^{\vee}\right)$, where $T^{\vee}$ is a torus of $G^{\vee}$, and $\sigma^{*}, \sigma^{\circ}$ induce automorphisms on $G^{\vee}$ and $T^{\vee}$, respectively. Since $\sigma^{*}$ and $\sigma^{\circ}$ are compatible with $F$, we have $\bar{\sigma}$ preserves the torus $T^{\vee}$, i.e., $\bar{\sigma}\left(T^{\vee}\right)=T^{\vee}$. It induces the action of $\sigma$ on $X^{*}\left(T^{\vee}\right)$.

Let $B^{\vee}$ be the maximal subgroup of $G^{\vee}$, which stabilizes the highest weight line $V_{\lambda}(\lambda)$ in $V(\lambda)$, for any $\sigma$-invariant dominant weight $\lambda$ of $G^{\vee}$. It is easy to see $B^{\vee}$ is a Borel subgroup of $G$, and contains $T^{\vee}$. For any $\sigma$-invariant dominant weight $\lambda, \sigma$ acts on $V(\lambda)$ by interchanging MV cycles, especially $\sigma$ acts trivially on $V_{\lambda}(\lambda)$. From Lemma 4.1 and the triviality of $\sigma$ on $V_{\lambda}(\lambda)$, we have $\bar{\sigma}(b) \cdot V_{\lambda}(\lambda)=\sigma\left(b \cdot V_{\lambda}(\lambda)\right)=$ $\sigma\left(V_{\lambda}(\lambda)\right)=V_{\lambda}(\lambda)$, for any $b \in B^{\vee}$. Hence we have $\bar{\sigma}\left(B^{\vee}\right)=B^{\vee}$.

The coroots of $G \alpha_{i}^{\vee}, i \in I$, can be viewed as the roots of $G^{\vee}$, and $\sigma$ sends the root $\alpha_{i}^{\vee}$ to $\alpha_{\sigma(i)}^{\vee}$ automatically, since under the identification of $X^{*}\left(T^{\vee}\right)$ and $X_{*}$, the actions of $\sigma$ are compatible.

Since $\sigma\left(T^{\vee}\right)=T^{\vee}$ and $\sigma\left(B^{\vee}\right)=B^{\vee}$, we can see that $\sigma$ maps the root subgroup $U_{\alpha^{\vee}}$ to $U_{\sigma\left(\alpha^{\vee}\right)}$, where $\alpha^{\vee}$ is a root of $G^{\vee}$. In particular, $\sigma\left(U_{\alpha_{i}^{\vee}}\right)=U_{\alpha_{\sigma(i)}^{\vee}}$, for any $i \in I$.

Let $\mathscr{G}^{\vee}$ be the Lie algebra of $G^{\vee}$. Let $\tau$ be the automorphism on $\mathscr{G}^{\vee}$ induced from $\bar{\sigma}$. From the following Lemma 4.3. we know $\tau$ acts trivially on the simple root space $\mathscr{G}_{\alpha_{i}^{\vee}}^{\vee}$ and $\mathscr{G}_{-\alpha_{i}^{\vee}}^{\vee}$, for $i$ fixed by $\sigma$. Lift $\tau$ to $\bar{\sigma}$ on $G^{\vee}$, then $\bar{\sigma}$ acts trivially on the root subgroup $U_{\alpha_{i}^{\vee}}$ and $U_{-\alpha_{i}^{\vee}}$, for $i, \sigma(i)=i$. Hence we are able to find root subgroup homomorphisms $x_{i}^{\vee}: \mathbb{C} \rightarrow G^{\vee}$ and $y_{i}^{\vee}: \mathbb{C} \rightarrow G^{\vee}$, corresponding to $\alpha_{i}^{\vee}$ and $-\alpha_{i}^{\vee}$, such that $\bar{\sigma}\left(x_{i}^{\vee}(a)\right)=x_{\sigma(i)}^{\vee}(a)$ and $\bar{\sigma}\left(y_{i}^{\vee}(a)\right)=y_{\sigma(i)}^{\vee}(a)$, for any $a \in \mathbb{C}$, and for any $i \in I$.

Hence $\bar{\sigma}$ is a Dynkin automorphism with respect to a pinning $\left(G^{\vee}, T^{\vee}, B^{\vee}, x_{i}^{\vee}, y_{i}^{\vee}\right.$, $i \in I)$ of $G^{\vee}$.

Assume the highest root is $\gamma^{\vee}$, then it is $\sigma$-invariant. $\mathscr{G}^{\vee}$ admits a highest representation of $G^{\vee}$ with highest weight $\gamma^{\vee}$. Assume $e_{\alpha^{\vee}}$ is the basis corresponding to the unique MV cycle in the root space $\mathscr{G}_{\alpha^{\vee}}^{\vee}$, for each root $\alpha^{\vee}$ of $G^{\vee}$. By interchanging MV cycles, we get a linear operator $\sigma$ on $\mathscr{G}^{\vee}$, especially $\sigma\left(e_{\alpha^{\vee}}\right)=e_{\sigma\left(\alpha^{\vee}\right)}$. Recall $\tau$ is an automorphism on $\mathscr{G}^{\vee}$, we have

Lemma 4.3. As linear operators on $\mathscr{G}^{\vee}$, if $G^{\vee}$ is of type $A_{2 n}$, then $\tau=-\sigma$; otherwise $\tau=\sigma$.

Proof. Let $\mathscr{H}^{\vee}$ be the Lie algebra of $T^{\vee}$. It is a Cartan subalgebra of $\mathscr{G}^{\vee}$, and it can be identified with $X^{*} \otimes \mathbb{C}$, where the actions of $\tau$ on $\mathscr{H}^{\vee}$ and $\sigma$ on $X^{*}$ are compatible.

From Lemma 4.1 we have $\sigma([a, b])=[\tau(a), \sigma(b)]$, for two arbitrary elements $a$ and $b$ in $\mathscr{G}^{\vee}$. By Schur's lemma, we have $\tau=c \cdot \sigma$, for some nonzero constant $c$. 
Let $\gamma$ be the corresponding coroot of highest root $\gamma^{\vee}$, so it is $\sigma$-invariant. Since $\left[e_{\gamma^{\vee}}, e_{-\gamma^{\vee}}\right] \in \mathbb{C} \cdot \gamma$, we have $\left[e_{\gamma^{\vee}}, e_{-\gamma^{\vee}}\right]=\tau\left(\left[e_{\gamma^{\vee}}, e_{-\gamma^{\vee}}\right]\right)=\left[\tau\left(e_{\gamma^{\vee}}\right), \tau\left(e_{-\gamma^{\vee}}\right)\right]=$ $c^{2} \cdot\left[e_{\gamma^{\vee}}, e_{-\gamma^{\vee}}\right]$. Hence $c^{2}=1$.

If $G^{\vee}$ is of type $A_{2 n}$, there exists two adjacent simple roots $\alpha_{i}^{\vee}$ and $\alpha_{j}^{\vee}$, such that $\sigma(i)=j$, for $i$ and $j \in I$. Then we have $\tau\left(\left[e_{\alpha_{i}^{\vee}}, e_{\alpha_{j}^{\vee}}\right]\right)=\left[e_{\alpha_{j}^{\vee}}, e_{\alpha_{i}^{\vee}}\right]=-\left[e_{\alpha_{i}^{\vee}}, e_{\alpha_{j}^{\vee}}\right]$. Since $\alpha_{i}^{\vee}+\alpha_{j}^{\vee}$ is also $\sigma$-invariant, it forces $c=-1$.

If $G^{\vee}$ is of another type, then let $h_{i}=\left[e_{\alpha_{i}^{\vee}}, e_{-\alpha_{i}^{\vee}}\right]$. Then $\left\{h_{i}\right\}_{i \in I}$ is a basis of $\mathscr{H}^{\vee}$. Since $\sigma\left(\left[e_{\alpha_{i}^{\vee}}, e_{-\alpha_{i}^{\vee}}\right]\right)=\left[\tau\left(e_{\alpha_{i}^{\vee}}\right), \sigma\left(e_{-\alpha_{i}^{\vee}}\right)\right]=c \cdot\left[e_{\alpha_{\sigma(i)}^{\vee}}, e_{\alpha_{-\sigma(i)}^{\vee}}\right]$, we have $\sigma\left(h_{i}\right)=c \cdot h_{\sigma(i)}$. It is easy to see that $\operatorname{trace}\left(\left.\sigma\right|_{\mathscr{H} \vee}\right)=c \cdot \sharp\{i \in I \mid \sigma(i)=i\}$. Since there exists $i \in I$, such that $\sigma(i)=i$, when $G^{\vee}$ is not of type $A_{2 n}$, we have $\operatorname{trace}\left(\left.\sigma\right|_{\mathscr{H}^{\vee}}\right) \neq 0$. Moreover, $\sigma$ interchanges MV cycles in $\mathscr{H}^{\vee}$, so $\operatorname{trace}\left(\left.\tau\right|_{\mathscr{H}^{\vee}}\right) \geq 0$. We thus have $c=1$.

Remark 4.1. We can give another construction of the Dynkin automorphism on $G^{\vee}$ which is compatible with the action of $\sigma$ on MV cycles, by using Vasserot's explicit construction of the action of the dual group on cohomology of perverse sheaves $[\mathrm{V}]$. Moreover, this automorphism coincides with the one from Tannakian formalism.

We have shown that $\bar{\sigma}$ is a Dynkin automorphism, and from Lemma 4.1, we see that the twining character $\operatorname{ch}^{\sigma}(V(\lambda))=\sum_{\mu \in P(\lambda)^{\sigma}} \operatorname{trace}\left(\left.\sigma\right|_{V_{\mu}(\lambda)}\right) e^{\mu}$, where $\lambda$ is $\sigma$-invariant.

\section{Proposition 4.4.}

$$
\operatorname{ch}^{\sigma}(V(\lambda))=\frac{\sum_{w \in W^{\sigma}}(-1)^{\ell_{\sigma}(w)} e^{w(\lambda+\rho)}}{\sum_{w \in W^{\sigma}}(-1)^{\ell_{\sigma}(w)} e^{w(\rho)}} .
$$

Proof. Let $V^{\sigma}(\lambda)$ be the irreducible representation of $\left(G^{\sigma}\right)^{\vee}$ with highest weight $\lambda$. By the Weyl character formula for $G^{\sigma}$, we have

$$
\sum_{\mu \in P(\lambda)^{\sigma}} \operatorname{dim} V_{\mu}^{\sigma}(\lambda) e^{\mu}=\frac{\sum_{w \in W^{\sigma}}(-1)^{\ell_{\sigma}(w)} e^{w(\lambda+\rho)}}{\sum_{w \in W^{\sigma}}(-1)^{\ell_{\sigma}(w)} e^{w(\rho)}} .
$$

Comparing with our definition of twining character for $G^{\vee}$, we see that it is equivalent to showing that $\operatorname{trace}\left(\left.\sigma\right|_{V_{\mu}(\lambda)}\right)=\operatorname{dim} V_{\mu}^{\sigma}(\lambda)$, for any $\mu \in P(\lambda)^{\sigma}$. By Lemma 4.1, $\operatorname{trace}\left(\left.\sigma\right|_{V_{\mu}(\lambda)}=\sharp\left(\operatorname{MVC}_{G}^{\mu}(\lambda)^{\sigma}\right)\right.$. Hence our proposition follows from Theorem 3.16

\section{REFERENCES}

[A] J. Anderson, A polytope calculus for semisimple groups, Duke Math. J. 116 (2003), 567588. MR.1958098 (2004a:20047)

[DM] P. Deligne and J. Milne, Tannakian categories in "Hodge cycles and motives", Springer, Lecture Notes, 900 (1982), 101-228.

[G] V. Ginzburg, Perverse sheaves on a loop group and Langlands duality, preprint arXiv:alggeom/951107.

[J] J. C. Jantzen, Darstellungen halbeinfacher algebraischer Gruppen und zugeordnete kontravariante Formen, Bonner Math. Schriften 67 (1973). MR0401935 (53:5761)

[K1] J. Kamnitzer, Mirković-Vilonen cycles and polytopes, preprint arXiv:math.AG/0501365.

[KLP] S. Kumar, G. Lusztig and D. Prasad, Characters of simply-laced nonconnected groups versus characters of nonsimply-laced connected groups, preprint arXiv: math.RT/0701615.

[L1] G. Lusztig, Total positivity in reductive groups, Lie theory and geometry, Progress in Math. 123, Birkhäuser Boston, (1994) MR1327548 (96m:20071) 
[MV] I. Mirković and K. Vilonen, Geometric Langlands duality and representations of algebraic groups over commutative rings, Ann. of Math. 166 (2007), no. 1, 95-143. MR2342692 (2008m:22027)

[N] S. Naito, Twining character formula of Borel-Weil-Bott Type, J. Math. Sci. Univ. Tokyo, 9 (2002), 637-658. MR.1947485 (2003m:20061)

[NS1] S. Naito and D. Sagaki, Action of a diagram automorphism on Mirković-Vilonen polytopes, a talk given at a conference in Karuizawa, June, 2007.

[NS2] S. Naito and D. Sagaki, A modification of the Anderson-Mirković conjecture for MirkovićVilonen polytopes in types $B$ and $C$, J. Algebra 320 (2008), no. 1, 387-416. MR 2417995

[ST] R. Steinberg, Endomorphism of linear algebraic groups. Memoirs of the American Mathematical Society, No. 80, American Mathematical Society, Providence, RI. MR 0230728 $(37: 6288)$

[V] E. Vasserot, On the action of the dual group on the cohomology of perverse sheaves on the affine Grassmannian, Compositio Math. 131 (2002), no. 1, 51-60. MR1895920 (2003b:20062)

Academy of Mathematics and Systems Science, Chinese Academy of Sciences, Beijing 100080, China

Current address: School of Mathematical Sciences, Tel Aviv University, Ramat Aviv, Tel Aviv 69978, Israel

E-mail address: hjzzjh@gmail.com 